\title{
Global Stability Analysis for Periodic Solution in Discontinuous Neural Networks with Nonlinear Growth Activations
}

\author{
Yingwei $\mathrm{Li}^{1}$ and Huaiqin $\mathrm{Wu}^{2}$ \\ ${ }^{1}$ College of Information Science and Engineering, Yanshan University, Qinhuangdao 066004, China \\ ${ }^{2}$ Department of Applied Mathematics, Yanshan University, Qinhuangdao 066004, China \\ Correspondence should be addressed to Huaiqin Wu, huaiqinwu@ysu.edu.cn
}

Received 30 December 2008; Accepted 18 March 2009

Recommended by Toka Diagana

This paper considers a new class of additive neural networks where the neuron activations are modelled by discontinuous functions with nonlinear growth. By Leray-Schauder alternative theorem in differential inclusion theory, matrix theory, and generalized Lyapunov approach, a general result is derived which ensures the existence and global asymptotical stability of a unique periodic solution for such neural networks. The obtained results can be applied to neural networks with a broad range of activation functions assuming neither boundedness nor monotonicity, and also show that Forti's conjecture for discontinuous neural networks with nonlinear growth activations is true.

Copyright (C) 2009 Y. Li and H. Wu. This is an open access article distributed under the Creative Commons Attribution License, which permits unrestricted use, distribution, and reproduction in any medium, provided the original work is properly cited.

\section{Introduction}

The stability of neural networks, which includes the stability of periodic solution and the stability of equilibrium point, has been extensively studied by many authors so far; see, for example, [1-15]. In [1-4], the authors investigated the stability of periodic solutions of neural networks with or without time delays, where the assumptions on neuron activation functions include Lipschitz conditions, bounded and/or monotonic increasing property. Recently, in [13-15], the authors discussed global stability of the equilibrium points for the neural networks with discontinuous neuron activations. Particularly, in [14], Forti conjectures that all solutions of neural networks with discontinuous neuron activations converge to an asymptotically stable limit cycle whenever the neuron inputs are periodic functions. As far as we know, there are only works of Wu in [5, 7] and Papini and Taddei in [9] dealing with this 
conjecture. However, the activation functions are required to be monotonic in $[5,7,9]$ and to be bounded in [5, 7].

In this paper, without assumptions of the boundedness and the monotonicity of the activation functions, by the Leray-Schauder alternative theorem in differential inclusion theory and some new analysis techniques, we study the existence of periodic solution for discontinuous neural networks with nonlinear growth activations. By constructing suitable Lyapunov functions we give a general condition on the global asymptotical stability of periodic solution. The results obtained in this paper show that Forti's conjecture in [14] for discontinuous neural networks with nonlinear growth activations is true.

For later discussion, we introduce the following notations.

Let $x=\left(x_{1}, \ldots, x_{n}\right)^{\prime}, y=\left(y_{1}, \ldots, y_{n}\right)^{\prime}$, where the prime means the transpose. By $x>0$ (resp., $x \geq 0$ ) we mean that $x_{i}>0$ (resp., $x_{i} \geq 0$ ) for all $i=1, \ldots, n .\|x\|=\left(\sum_{i=1}^{n} x_{i}^{2}\right)^{1 / 2}$ denotes the Euclidean norm of $x .\langle x, y\rangle=\sum_{i=1}^{n} x_{i} y_{i}\langle\cdot, \cdot\rangle$ denotes the inner product. $\|B\|$ denotes 2norm of matrix $B \in R^{n \times n}$, that is, $\|B\|=\sqrt{\sigma\left(B^{\prime} B\right)}$, where $\sigma\left(B^{\prime} B\right)$ denotes the spectral radius of $B^{\prime} B$.

Given a set $C \subset R^{n}$, by $K[C]$ we denote the closure of the convex hull of $C$, and $P_{k c}(C)$ denotes the collection of all nonempty, closed, and convex subsets of $C$. Let $X$ be a Banach space, and $\|x\|_{X}$ denotes the norm of $x, \forall x \in X$. By $L^{1}\left([0, \omega], R^{n}\right)$ we denote the Banach space of the Lebesgue integrable functions $x(\cdot):[0, \omega] \rightarrow R^{n}$ equipped with the norm $\int_{0}^{\omega}\|x(t)\| d t$. Let $V: R^{n} \rightarrow R$ be a locally Lipschitz continuous function. Clarke's generalized gradient [16] of $V$ at $x$ is defined by

$$
\partial V(x)=K\left[\left\{\lim _{i \rightarrow \infty} \nabla V\left(x_{i}\right): \lim _{i \rightarrow \infty} x_{i}=x, x_{i} \in R^{n} \backslash \Omega_{V} \cup \mathcal{N}\right\}\right],
$$

where $\Omega_{V} \subset R^{n}$ is the set of Lebesgue measure zero where $\nabla V$ does not exist, and $N \subset R^{n}$ is an arbitrary set with measure zero.

The rest of this paper is organized as follows. Section 2 develops a discontinuous neural network model with nonlinear growth activations, and some preliminaries also are given. Section 3 presents the proof on the existence of periodic solution. Section 4 discusses global asymptotical stability of the neural network. Illustrative examples are provided to show the effectiveness of the obtained results in Section 5.

\section{Model Description and Preliminaries}

The model we consider in the present paper is the neural networks modeled by the differential equation

$$
\dot{x}(t)=-A x(t)+B g(x(t))+I(t)
$$

where $x(t)=\left(x_{1}(t), \ldots, x_{n}(t)\right)^{\prime}$ is the vector of neuron states at time $t ; A$ is an $n \times n$ matrix representing the neuron inhibition; $B$ is an $n \times n$ neuron interconnection matrix; $g: R^{n} \rightarrow R^{n}$, $g_{i}, \quad i=1, \ldots, n$, represents the neuron input-output activation and $I(t)=\left(I_{1}(t), \ldots, I_{n}(t)\right)^{\prime}$ is the continuous $\omega$-periodic vector function denoting neuron inputs.

Throughout the paper, we assume that

$H_{1}$ : (1) $g_{i}$ has only a finite number of discontinuity points in every compact set of $R$. Moreover, there exist finite right limit $g_{i}\left(v_{k}^{+}\right)$and left limit $g_{i}\left(v_{k}^{-}\right)$at discontinuity point $v_{k}$. 
(2) $g$ has the nonlinear growth property, that is, for all $x \in R^{n}$

$$
\|K[g(x)]\|=\sup _{\gamma \in K[g(x)]}\|\gamma\| \leq c\left(1+\|x\|^{\alpha}\right)
$$

where $c>0, \alpha \in(0,1)$ are constants, and $K[g(x)]=\left(K\left[g_{1}\left(x_{1}\right)\right], \ldots, K\left[g_{n}\left(x_{n}\right)\right]\right)^{\prime}$.

$H_{2}:\langle x, A x\rangle \geq \rho\|x\|^{2}$ for all $x \in R^{n}$, where $\rho>0$ is a constant.

Under the assumption $H_{1}, g$ is undefined at the points where $g$ is discontinuous. Equation (2.1) is a differential equation with a discontinuous right-hand side. For (2.1), we adopt the following definition of the solution in the sense of Filippov [17] in this paper.

Definition 2.1. Under the assumption $H_{1}$, a solution of (2.1) on an interval $[0, b)$ with the initial value $x(0)=x_{0}$ is an absolutely continuous function satisfying

$$
\dot{x}(t) \in-A x(t)+B K[g(x(t))]+I(t), \quad \text { for a.e. } t \in[0, b) .
$$

It is easy to see that $\phi:(x, t) \hookrightarrow-A x+B K[g(x)]+I(t)$ is an upper semicontinuous set-valued map with nonempty compact convex values; hence, it is measurable [18]. By the measurable selection theorem [19], if $x(\cdot)$ is a solution of (2.1), then there exists a measurable function $\eta(t) \in K[g(x(t))]$ such that

$$
\dot{x}(t)=-A x(t)+B \eta(t)+I(t), \quad \text { for a.e. } t \in[0, b) .
$$

Consider the following differential inclusion problem

$$
\begin{gathered}
\dot{x}(t) \in-A x(t)+B K[g(x(t))]+I(t), \quad \text { for a.e. } t \in[0, \omega], \\
x(0)=x(\omega) .
\end{gathered}
$$

It easily follows that if $x(t)$ is a solution of (2.5), then $x^{*}(t)$ defined by

$$
x^{*}(t)=x(t-j \omega), \quad t \in[j \omega,(j+1) \omega], \quad j \in N
$$

is an $\omega$-periodic solution of (2.1). Hence, for the neural network (2.1), finding the periodic solutions is equivalent to finding solutions of (2.5).

Definition 2.2. The periodic solution $x^{*}(t)$ with initial value $x^{*}(0)=x_{0}^{*}$ of the neural network (2.1) is said to be globally asymptotically stable if $x^{*}$ is stable and for any solution $x(t)$, whose existence interval is $[0,+\infty)$, we have $\lim _{t \rightarrow+\infty}\left\|x(t)-x^{*}(t)\right\|=0$.

Lemma 2.3. If $X$ is a Banach space, $C \subseteq X$ is nonempty closed convex with $0 \in C$ and $G: C \rightarrow$ $P_{k c}(C)$ is an upper semicontinuous set-valued map which maps bounded sets into relatively compact sets, then one of the following statements is true:

(a) the set $\Gamma=\{x \in C: x \in \lambda G(x), \lambda \in(0,1)\}$ is unbounded;

(b) the $G(\cdot)$ has a fixed point in $C$, that is, there exists $x \in C$, such that $x \in G(x)$. 
Lemma 2.3 is said to be the Leray-Schauder alternative theorem, whose proof can be found in [20]. Define the following:

$$
\begin{gathered}
W^{1,1}\left([0, \omega], R^{n}\right)=\{x(\cdot): x(\cdot) \text { is absolute continuous on }[0, \omega]\}, \\
W_{p}^{1,1}\left([0, \omega], R^{n}\right)=\left\{x(\cdot) \in W^{1,1}\left([0, \omega], R^{n}\right) \mid x(0)=x(\omega)\right\}, \\
\|x\|_{W^{1,1}}=\int_{0}^{\omega}\|x(t)\| d t+\int_{0}^{\omega}\|\dot{x}(t)\| d t, \quad \forall x \in W^{1,1}\left([0, \omega], R^{n}\right),
\end{gathered}
$$

then $\|\cdot\|_{W^{1,1}}$ is a class of norms of $W^{1,1}\left([0, \omega], R^{n}\right), W^{1,1}\left([0, \omega], R^{n}\right)$, and $W_{p}^{1,1}\left([0, \omega], R^{n}\right) \subset$ $W^{1,1}\left([0, \omega], R^{n}\right)$ are Banach space under the norm $\|x\|_{W^{1,1}}$.

If $V: R^{n} \rightarrow R$ is (i) regular in $R^{n}$ [16]; (ii) positive definite, that is, $V(x)>0$ for $x \neq 0$, and $V(0)=0$; (iii) radially unbounded, that is, $V(x) \rightarrow+\infty$ as $\|x\| \rightarrow+\infty$, then $V(x)$ is said to be $\mathrm{C}$-regular.

Lemma 2.4 (Chain Rule [15]). If $V(x)$ is C-regular and $x(\cdot):[0,+\infty) \rightarrow R^{n}$ is absolutely continuous on any compact interval of $[0,+\infty)$, then $x(t)$ and $V(x(t)):[0,+\infty) \rightarrow R$ are differential for a.e. $t \in[0,+\infty)$, and one has

$$
\frac{d}{d t} V(x(t))=\left\langle\zeta, \frac{d x(t)}{d t}\right\rangle, \quad \forall \zeta \in \partial V(x)
$$

\section{Existence of Periodic Solution}

Theorem 3.1. If the assumptions $H_{1}$ and $H_{2}$ hold, then for any $x_{0} \in R^{n},(2.1)$ has at least a solution defined on $[0,+\infty)$ with the initial value $x(0)=x_{0}$.

Proof. By the assumption $H_{1}$, it is easy to get that $\phi:(x, t) \hookrightarrow-A x+B K[g(x)]+I(t)$ is an upper semicontinuous set-valued map with nonempty, compact, and convex values. Hence, by Definition 2.1, the local existence of a solution $x(\cdot)$ for $(2.1)$ on $\left[0, t_{0}\right], t_{0}>0$, with $x(0)=x_{0}$, is obvious [17].

Set $\psi(t, x)=B K[g(x)]+I(t)$. Since $I(\cdot)$ is a continuous $\omega$-periodic vector function, $I(\cdot)$ is bounded, that is, there exists a constant $\supset>0$ such that $\|I(t)\| \leq \supset, t \in[0, \omega]$. By the assumption $H_{1}$, we have

$$
\sup _{x \in R^{n}, t \in[0,+\infty]}\|\psi(t, x)\| \leq\|B\|\|K[g(x)]\|+\|I(t)\| \leq c\|B\|\left(1+\|x\|^{\alpha}\right)+\supset .
$$

By $\lim _{\|x\| \rightarrow+\infty}\left(c\|B\|\left(1+\|x\|^{\alpha}\right)+\supset\right) /\|x\|=0$, we can choose a constant $\widetilde{R}>0$, such that when $\|x\|>\widetilde{R}$

$$
\frac{c\|B\|\left(1+\|x\|^{\alpha}\right)+\supset}{\|x\|}<\frac{\rho}{2} .
$$


By (2.4), (3.1), (3.2), and the Cauchy inequality, when $\|x(t)\|>\widetilde{R}$,

$$
\begin{aligned}
\frac{1}{2} \frac{d}{d t}\|x(t)\|^{2} & =\langle x(t), \dot{x}(t)\rangle=\langle x(t),-A x(t)+B \eta(t)+I(t)\rangle \\
& =-\langle x(t), A x(t)\rangle+\langle x(t), B \eta(t)+I(t)\rangle \\
& \leq-\rho\|x(t)\|^{2}+\|x(t)\|\left(c\|B\|\left(1+\|x(t)\|^{\alpha}\right)+\supset\right) \\
& =\left(-\rho+\frac{c\|B\|\left(1+\|x(t)\|^{\alpha}\right)+\supset}{\|x(t)\|}\right)\|x(t)\|^{2} \\
& <-\frac{\rho}{2}\|x(t)\|^{2}<0 .
\end{aligned}
$$

Therefore, let $\bar{R}=\max \left\{\left\|x_{0}\right\|, \widetilde{R}\right\}$, then, by (3.3), it follows that $\|x(t)\| \leq \bar{R}$ on $\left[0, t_{0}\right]$. This means that the local solution $x(\cdot)$ is bounded. Thus, (2.1) has at least a solution with the initial value $x(0)=x_{0}$ on $[0,+\infty)$. This completes the proof.

Theorem 3.1 shows the existence of solutions of (2.1). In the following, we will prove that (2.1) has an $\omega$-periodic solution.

Let $L x=\dot{x}+A x$ for all $x \in W_{p}^{1,1}\left([0, \omega], R^{n}\right)$, then $L: W_{p}^{1,1}\left([0, \omega], R^{n}\right) \rightarrow L^{1}\left([0, \omega], R^{n}\right)$ is a linear operator.

Proposition 3.2. $L: W_{p}^{1,1}\left([0, \omega], R^{n}\right) \rightarrow L^{1}\left([0, \omega], R^{n}\right)$ is bounded, one to one and surjective.

Proof. For any $x \in W_{p}^{1,1}\left([0, \omega], R^{n}\right)$, we have

$$
\begin{aligned}
\|L x\|_{L^{1}} & =\int_{0}^{\omega}\|\dot{x}(t)+A x(t)\| d t \leq \int_{0}^{\omega}\|\dot{x}(t)\| d t+\int_{0}^{\omega}\|A x(t)\| d t \\
& \leq \int_{0}^{\omega}\|\dot{x}(t)\| d t+\|A\| \int_{0}^{\omega}\|x(t)\| d t \leq \max \{1,\|A\|\}\|x\|_{W^{1,1}}
\end{aligned}
$$

this implies that $L$ is bounded.

Let $x^{1}, x^{2} \in W_{p}^{1,1}\left([0, \omega], R^{n}\right)$. If $L x^{1}=L x^{2}$, then

$$
\dot{x}^{1}(t)-\dot{x}^{2}(t)=-A\left(x^{1}(t)-x^{2}(t)\right) .
$$

By the assumption $\mathrm{H}_{2}$,

$$
\begin{aligned}
& \frac{d}{d t}\left(-\left\|x^{1}(t)-x^{2}(t)\right\|^{2}\right)=-2\left\langle x^{1}(t)-x^{2}(t), \dot{x}^{1}(t)-\dot{x}^{2}(t)\right\rangle \\
& \quad=2\left\langle x^{1}(t)-x^{2}(t), A\left[x^{1}(t)-x^{2}(t)\right]\right\rangle \geq 2 \rho\left\|x^{1}(t)-x^{2}\right\|^{2}
\end{aligned}
$$


Noting $x^{1}(0)=x^{1}(\omega), x^{2}(0)=x^{2}(\omega)$, we have

$$
\int_{0}^{\omega} \frac{d}{d t}\left(-\left\|x^{1}(t)-x^{2}(t)\right\|^{2}\right) d t=\left\|x^{1}(0)-x^{2}(0)\right\|^{2}-\left\|x^{1}(\omega)-x^{2}(\omega)\right\|^{2}=0,
$$

By (3.6),

$$
0 \leq \int_{0}^{\omega} 2 \rho\left\|x^{1}(t)-x^{2}(t)\right\|^{2} d t \leq \int_{0}^{\omega} \frac{d}{d t}\left(-\left\|x^{1}(t)-x^{2}(t)\right\|^{2}\right) d t=0
$$

Hence $\left\|x^{1}-x^{2}\right\|=0$. It follows $x^{1}=x^{2}$. This shows that $L$ is one to one.

Let $f(\cdot) \in L^{1}\left([0, \omega], R^{n}\right)$. In order to verify that $L$ is surjective, in the following, we will prove that there exists $x(\cdot) \in W_{p}^{1,1}\left([0, \omega], R^{n}\right)$ such that

$$
L x=f,
$$

that is, we will prove that there exists a solution for the differential equation

$$
\begin{gathered}
\dot{x}(t)=-A x(t)+f(t), \\
x(0)=x(\omega) .
\end{gathered}
$$

Consider Cauchy problem

$$
\begin{gathered}
\dot{x}(t)=-A x(t)+f(t), \\
x(0)=\xi .
\end{gathered}
$$

It is easily checked that

$$
x(t)=e^{-A t}\left(\xi+\int_{0}^{t} f(s) e^{A s} d s\right)
$$

is the solution of (3.11). By (3.12), we want $\xi=x(\omega)$, then

$$
e^{-A \omega}\left(\xi+\int_{0}^{\omega} f(s) e^{A s} d s\right)=\xi
$$

that is,

$$
\left(I-e^{-A \omega}\right) \xi=e^{-A \omega} \int_{0}^{\omega} f(s) e^{A s} d s
$$


By the assumption $H_{2}, I-e^{-A \omega}$ is a nonsingular matrix, where $I$ is a unit matrix. Thus, by (3.14), if we take $\xi$ as

$$
\xi=\left(I-e^{-A \omega}\right)^{-1} e^{-A \omega} \int_{0}^{\omega} f(s) e^{A s} d s
$$

in (3.12), then (3.12) is the solution of (3.10). This shows that $L$ is surjective. This completes the proof.

By the Banach inverse operator theorem, $L^{-1}: L^{1}\left([0, \omega], R^{n}\right) \rightarrow W_{p}^{1,1}\left([0, \omega], R^{n}\right) \subseteq$ $L^{1}\left([0, \omega], R^{n}\right)$ is a bounded linear operator.

For any $x(\cdot) \in L^{1}\left([0, \omega], R^{n}\right)$, define the set-valued map $N$ as

$$
\mathcal{N}(x)=\left\{v(\cdot) \in L^{1}\left([0, \omega], R^{n}\right) \mid v(t) \in \psi(t, x(t)), \text { for a.e. } t \in[0, \omega]\right\} .
$$

Then $\mathcal{N}$ has the following properties.

Proposition 3.3. $\mathcal{N}(\cdot): L^{1}\left([0, \omega], R^{n}\right) \hookrightarrow 2^{L^{1}\left([0, \omega], R^{n}\right)_{w}}$ has nonempty closed convex values in $L^{1}\left([0, \omega], R^{n}\right)$ and is also upper semicontinuous from $L^{1}\left([0, \omega], R^{n}\right)$ into $L^{1}\left([0, \omega], R^{n}\right)$ endowed with the weak topology.

Proof. The closedness and convexity of values of $\mathcal{N}(\cdot)$ are clear. Next, we verify the nonemptiness. In fact, for any $x \in L^{1}\left([0, \omega], R^{n}\right)$, there exists a sequence of step functions $\left\{s_{n}\right\}_{n \geq 1}$ such that $s_{n}(t) \rightarrow x(t)$ and $\left\|s_{n}(t)\right\| \leq\|x(t)\|$ a.e. on $[0, \omega]$. By the assumption $H_{1}(1)$ and the continuity of $I(t)$, we can get that $(t, x) \rightarrow \psi(t, x)$ is graph measurable. Hence, for any $n \geq 1, t \rightarrow \psi\left(t, s_{n}(t)\right)$ admits a measurable selector $f_{n}(t)$. By the assumption $H_{1}(2)$, $\left\{f_{n}(\cdot)\right\}_{n \geq 1}$ is uniformly integrable. So by Dunford-Pettis theorem, there exists a subsequence $\left\{f_{n_{k}}(\cdot)\right\}_{k \geq 1} \subseteq\left\{f_{n}(\cdot)\right\}_{n \geq 1}$ such that $f_{n_{k}} \rightarrow f$ weakly in $L^{1}\left([0, \omega], R^{n}\right)$. Hence, from [21, Theorem 3.1], we have

$$
f(t) \in K\left[w-\varlimsup\left\{f_{n_{k}}(t)\right\}_{k \geq 1}\right] \subseteq K\left[w-\varlimsup \bar{l} \psi\left(t, s_{n_{k}}(t)\right)\right], \quad \text { a.e. on }[0, w]
$$

Noting that $\psi(t, \cdot)$ is an upper semicontinuous set-valued map with nonempty closed convex values on $x$ for a.e. $t \in[0, \omega], K\left[w-\varlimsup \overline{\lim } \psi\left(t, s_{n_{k}}(t)\right)\right] \subseteq \psi(t, x(t))$. Therefore, $f \in \mathcal{N}(x)$. This shows that $\mathcal{N}(x(\cdot))$ is nonempty.

At last we will prove that $\mathcal{N}(\cdot)$ is upper semicontinuous from $L^{1}\left([0, \omega], R^{n}\right)$ into $L^{1}\left([0, \omega], R^{n}\right)_{w}$. Let $C$ be a nonempty and weakly closed subset of $L^{1}\left([0, \omega], R^{n}\right)$, then we need only to prove that the set

$$
\mathcal{N}^{-1}(C)=\left\{x \in L^{1}\left([0, \omega], R^{n}\right): \mathcal{N}(x) \bigcap C \neq \emptyset\right\}
$$

is closed. Let $\left\{x_{n}\right\}_{n \geq 1} \subseteq \mathcal{N}^{-1}(C)$ and assume $x_{n} \rightarrow x$ in $L^{1}\left([0, \omega], R^{n}\right)$, then there exists a subsequence $\left\{x_{n_{k}}\right\}_{k \geq 1} \subseteq\left\{x_{n}\right\}_{n \geq 1}$ such that $x_{n_{k}}(t) \rightarrow x(t)$ a.e. on $[0, \omega]$. Take $f_{k} \in \mathcal{N}\left(x_{n_{k}}\right) \cap C$, $n \geq 1$, then By the assumption $H_{1}(2)$ and Dunford-Pettis theorem, there exists a subsequence 
$\left\{f_{k_{m}}(t)\right\}_{m \geq 1} \subseteq\left\{f_{k}(t)\right\}_{k \geq 1}$ such that $f_{k_{m}} \rightarrow f \in C$ weakly in $L^{1}\left([0, \omega], R^{n}\right)$. As before we have

$$
f(t) \in K\left[w-\varlimsup\left\{f_{k_{m}}(t)\right\}_{m \geq 1}\right] \subseteq K\left[w-\varlimsup \overline{\lim } \psi\left(t, x_{n_{k_{m}}}(t)\right)\right] \subseteq \psi(t, x(t)), \quad \text { a.e. on }[0, w]
$$

This implies $f \in \mathcal{N}(x) \cap C$, that is, $\mathcal{N}^{-1}(C)$ is closed in $L^{1}\left([0, \omega], R^{n}\right)$. The proof is complete.

Theorem 3.4. Under the assumptions $H_{1}$ and $H_{2}$, there exists a solution for the boundary-value problem (2.5), that is, the neural network (2.1) has an w-periodic solution.

Proof. Consider the set-valued map $L^{-1} \circ \mathcal{N}$. Since $L^{-1}$ is continuous and $\mathcal{N}$ is upper semicontinuous, the set-valued map $L^{-1} \circ \mathcal{N}$ is upper semicontinuous. Let $K \subset L^{1}\left([0, \omega], R^{n}\right)$ be a bounded set, then

$$
\mathcal{N}(K)=\bigcup_{x \in K} \mathcal{N}(x)
$$

is a bounded subset of $L^{1}\left([0, \omega], R^{n}\right)$. Since $L^{-1}$ is a bounded linear operator, $L^{-1}(\mathcal{N}(K))$ is a bounded subset of $W_{p}^{1,1}\left([0, \omega], R^{n}\right)$. Noting that $W_{p}^{1,1}\left([0, \omega], R^{n}\right)$ is compactly embedded in $L^{1}\left([0, \omega], R^{n}\right), L^{-1}(N(K))$ is a relatively compact subset of $L^{1}\left([0, \omega], R^{n}\right)$. Hence by Proposition 3.3, $L^{-1} \circ \mathcal{N}: L^{1}\left([0, \omega], R^{n}\right) \rightarrow P_{k c}\left(L^{1}\left([0, \omega], R^{n}\right)\right)$ is the upper semicontinuous set-valued map which maps bounded sets into relatively compact sets.

For any $r(x) \in K[g(x)]$, when $x \neq 0, \lambda \in(0,1)$, by (3.1) and the Cauchy inequality,

$$
\begin{aligned}
\langle x,-A x+\lambda B r(x)+\lambda I(t)\rangle & =-\langle x, A x\rangle+\langle x, \lambda B r(x)+\lambda I(t)\rangle \\
& \leq-\rho\|x\|^{2}+\lambda\|\psi(t, x)\|\|x\| \\
& \leq-\rho\|x\|^{2}+\left[c\|B\|\left(1+\|x\|^{\alpha}\right)+\supset\right]\|x\| \\
& =\left(-\rho+\frac{c\|B\|\left(1+\|x\|^{\alpha}\right)+\supset}{\|x\|}\right)\|x\|^{2} .
\end{aligned}
$$

Arguing as (3.2), we can choose a constant $K_{0}>0$, such that when $\|x\|>K_{0}$,

$$
\frac{c\|B\|\left(1+\|x\|^{\alpha}\right)+\supset}{\|x\|}<\frac{\rho}{2}
$$

Therefore, when $\|x\|>K_{0}$, by (3.21),

$$
\langle x,-A x+\lambda B r(x)+\lambda I(t)\rangle<-\frac{\rho}{2}\|x\|^{2}, \quad \forall r(x) \in K[g(x)] .
$$

Set

$$
\Gamma=\left\{x \in L^{1}\left([0, \omega], R^{n}\right): x \in \lambda L^{-1} \circ \mathcal{N}(x), \lambda \in(0,1)\right\}
$$


In the following, we will prove that $\Gamma$ is a bounded subset of $L^{1}\left([0, \omega], R^{n}\right)$. Let $x \in \Gamma$, then $x \in \lambda L^{-1} \circ \mathcal{N}(x)$, that is, $L x \in \mathcal{N}(x)$. By the definition of $\mathcal{N}(\cdot)$, there exists a measurable selection $v(t) \in K[g(x(t))]$, such that

$$
\dot{x}(t)+A x(t)=\lambda B v(t)+\lambda I(t) .
$$

By (3.23) and (3.25), $\max _{t \in[0, \omega]}\|x(t)\| \leq K_{0}$. Otherwise, $\max _{t \in[0, \omega]}\|x(t)\|>K_{0}$. By $L^{-1}$ : $L^{1}\left([0, \omega], R^{n}\right) \rightarrow W_{p}^{1,1}\left([0, \omega], R^{n}\right)$, we have $x(0)=x(\omega)$. Since $x(\cdot)$ is continuous, we can choose $t_{0} \in(0, \omega]$, such that

$$
\left\|x\left(t_{0}\right)\right\|=\max _{t \in[0, \omega]}\|x(t)\|>K_{0} .
$$

Thus, there exists a constant $\delta_{t_{0}}>0$, such that when $t \in\left(t_{0}-\delta_{t_{0}}, t_{0}\right],\|x(t)\|>K_{0}$. By (3.23) and (3.25),

$$
\begin{aligned}
& 0 \leq \frac{1}{2}\left\|x\left(t_{0}\right)\right\|^{2}-\frac{1}{2}\|x(t)\|^{2}=\frac{1}{2} \int_{t}^{t_{0}} \frac{d}{d s}\left(\|x(s)\|^{2}\right) d s=\int_{t}^{t_{0}}\langle x(s), \\
& \dot{x}(s)\rangle d s=\int_{t}^{t_{0}}\langle x(s), \\
& -A x(s)+\lambda B v(s)+\lambda I(s)\rangle d s<-\frac{\rho}{2} \int_{t}^{t_{0}}\|x(s)\|^{2} d s<0, \\
& \text { for } t \in\left(t_{0}-\delta_{t_{0}}, t_{0}\right] .
\end{aligned}
$$

This is a contradiction. Thus, for any $x \in \Gamma, \max _{t \in[0, \omega]}\|x(t)\| \leq K_{0}$. Furthermore, we have

$$
\|\mathrm{x}\|_{L^{1}}=\int_{0}^{\omega}\|x(s)\| d s \leq \omega K_{0}, \quad \forall x \in \Gamma .
$$

This shows that $\Gamma$ is a bounded subset of $L^{1}\left([0, \omega], R^{n}\right)$.

By Lemma 2.3, the set-valued map $L^{-1} \circ \mathcal{N}$ has a fixed point, that is, there exists $x^{*} \in$ $L^{1}\left([0, \omega], R^{n}\right)$ such that $x^{*} \in L^{-1} \circ \mathcal{N}\left(x^{*}\right), L x^{*} \in \mathcal{N}\left(x^{*}\right)$. Hence there exists a measurable selection $\eta^{*}(t) \in K\left[g\left(x^{*}(t)\right)\right]$, such that

$$
\dot{x}^{*}(t)+A x^{*}(t)=B \eta^{*}(t)+I(t) .
$$

By the definition of $L^{-1}, x^{*} \in W_{p}^{1,1}\left([0, \omega], R^{n}\right)$. Moreover, by Definition 2.1 and (3.29), $x^{*}(\cdot)$ is a solution of the boundary-value problem (2.5), that is, the neural network (2.1) has an $\omega$-periodic solution. The proof is completed.

\section{Global Asymptotical Stability of Periodic Solution}

Theorem 4.1. Suppose that $H_{1}$ and the following assumptions are satisfied. 
$H_{3}$ : for each $i \in 1, \ldots, n$, there exists a constant $l_{i}>0$, such that for all two different numbers $u, v \in R$, for all $\gamma_{i} \in K\left[g_{i}(u)\right]$ and for all $\zeta_{i} \in K\left[g_{i}(u)\right]$

$$
\frac{\gamma_{i}-\zeta_{i}}{u-v} \geq-l_{i}
$$

$H_{4}: A=\operatorname{diag}\left(a_{1}, \ldots, a_{n}\right)>0$ is a diagonal matrix, and there exists a positive diagonal matrix $P=\operatorname{diag}\left(p_{1}, \ldots, p_{2}\right)>0$ such that $P(-B)+\left(-B^{\prime}\right) P>0$ and

$$
l_{i} p_{i}\|B\|^{2}<\frac{1}{2} \lambda_{m} a
$$

where $\lambda_{m}$ is the minimum eigenvalues of symmetric matrix $P(-B)+\left(-B^{\prime}\right) P, a=\min _{1 \leq i \leq n} a_{i}$, for all $i=1, \ldots, n$. Then the neural network (2.1) has a unique $w$-periodic solution which is globally asymptotically stable.

Proof. By the assumptions $H_{3}$ and $H_{4}$, there exists a positive constant $\beta$ such that

$$
\frac{\gamma_{i}-\zeta_{i}}{u-v} \geq-\frac{1}{2 \beta p_{i}}
$$

for all $\gamma_{i} \in K\left[g_{i}(u)\right], \zeta_{i} \in K\left[g_{i}(u)\right], i=1, \ldots, n$, and

$$
\frac{\|B\|^{2}}{\beta \lambda_{m}}<a
$$

In fact, from (4.2), we have

$$
\frac{\|B\|^{2}}{a \lambda_{m}}<\frac{1}{2 l_{i} p_{i}}
$$

Choose $\beta \in\left(\|B\|^{2} / a \lambda_{m}, 1 / 2 l_{i} p_{i}\right)$, then $-l_{i}>-\left(1 / 2 \beta P_{i}\right)$, which implies that (4.3) holds from (4.1), and (4.4) is also satisfied. By the assumption $H_{4}$, it is easy to get that the assumption $H_{2}$ is satisfied. By Theorem 3.4, the neural network (2.1) has an $\omega$-periodic solution. Let $x^{*}(\cdot)$ be an $\omega$-periodic solution of the neural network (2.1). Consider the change of variables $z(t)=$ $x(t)-x^{*}(t)$, which transforms (2.4) into the differential equation

$$
\dot{z}(t)=-A z(t)+B \tilde{\eta}(t)
$$

where $\tilde{\eta}(t) \in K[G(z(t))], G(z)=\left(G_{1}\left(z_{1}\right), \ldots, G_{n}\left(z_{n}\right)\right)^{\prime}$, and $G_{i}\left(z_{i}\right)=g_{i}\left(z_{i}+x_{i}^{*}\right)-\eta_{i}^{*}, \eta_{i}^{*} \in$ $K\left[g\left(x^{*}\right)\right]$. Obviously, $z=0$ is a solution of (4.6).

Consider the following Lyapunov function:

$$
V(z)=z^{\prime} z+2 \beta \sum_{i=1}^{n} p_{i} \int_{0}^{z_{i}} G_{i}(\rho) d \rho
$$


By (4.3),

$$
z_{i}^{2}+2 \beta p_{i} \int_{0}^{z_{i}} G_{i}(\rho) d \rho \geq \frac{1}{2} z_{i}^{2}
$$

and thus $V \geq(1 / 2)\|z\|^{2}$. In addition, it is easy to check that $V$ is regular in $R^{n}$ and $V(0)=0$. This implies that $V$ is C-regular. Calculate the derivative of $V(z)$ along the solution $z(t)$ of (4.6). By Lemma 2.4, (4.3), and (4.4),

$$
\begin{aligned}
\frac{\mathrm{d} V(z)}{\mathrm{d} t}= & \left(2 z^{\prime}(t)+2 \beta \tilde{\eta}^{\prime}(t) P\right)(-A z(t)+B \tilde{\eta}(t)) \\
\leq & -z^{\prime}(t) A z(t)+2 z^{\prime}(t) B \tilde{\eta}(t)+2 \beta \tilde{\eta}^{\prime}(t) P B \tilde{\eta}(t) \\
\leq & -a z^{\prime}(t) z(t)+2 z^{\prime}(t) B \tilde{\eta}(t)-\beta \lambda_{m} \tilde{\eta}^{\prime}(t) \tilde{\eta}(t) \\
\leq & -a z^{\prime}(t) z(t)+\frac{1}{\beta \lambda_{m}} z^{\prime}(t) B^{\prime} B z(t) \\
& -\left(\frac{1}{\sqrt{\beta \lambda_{m}}} B z(t)-\sqrt{\beta \lambda_{m}} \tilde{\eta}^{\prime}(t)\right)^{\prime}\left(\frac{1}{\sqrt{\beta \lambda_{m}}} B z(t)-\sqrt{\beta \lambda_{m}} \tilde{\eta}^{\prime}(t)\right) \\
\leq & -\left(a-\frac{\|B\|^{2}}{\beta \lambda_{m}}\right)\|z(t)\|^{2} \\
= & -\delta\|z(t)\|^{2},
\end{aligned}
$$

where $\delta=a-\left(\|B\|^{2} / \beta \lambda_{m}\right)>0$. Thus, the solution $z=0$ of (4.6) is globally asymptotically stable, so is the periodic solution $x^{*}(\cdot)$ of the neural network (2.1). Consequently, the periodic solution $x^{*}(\cdot)$ is unique. The proof is completed.

Remark 4.2. (1) If $g_{i}$ is nondecreasing, then the assumption $H_{3}$ obviously holds. Thus the assumption $\mathrm{H}_{3}$ is more general. equation

(2) In [14], Forti et al. considered delayed neural networks modelled by the differential

$$
\dot{x}(t)=-D x(t)+B g(x(t))+B^{\tau} g(x(t-\tau))+I,
$$

where $D$ is a positive diagonal matrix, and $B^{\tau}$ is an $n \times n$ constant matrix which represents the delayed neuron interconnection. When $g_{i}$ satisfies the assumption $H_{1}(1)$ and is nondecreasing and bounded, [14] investigated the existence and global exponential stability of the equilibrium point, and global convergence in finite time for the neural network (4.10). At last, Forti conjectured that the neural network

$$
\dot{x}(t)=-D x(t)+B g(x(t))+B^{\tau} g(x(t-\tau))+I(t)
$$

has a unique periodic solution and all solutions converge to the asymptotically stable limit cycle when $I(\cdot)$ is a periodic function. When $B^{\tau}=0$, the neural network (4.11) changes as 


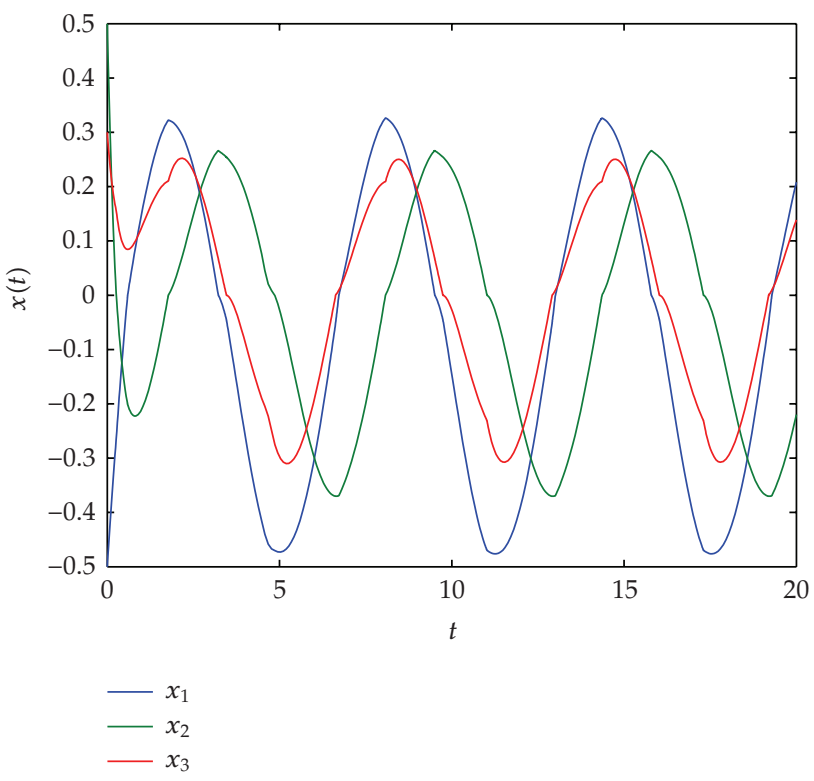

Figure 1: Time-domain behavior of the state variables $x_{1}, x_{2}$, and $x_{3}$.

the neural network (2.1) without delays. Thus, without assumptions of the boundedness and the monotonicity of the activation functions, Theorem 4.1 obtained in this paper shows that Forti's conjecture for discontinuous neural networks with nonlinear growth activations and without delays is true.

\section{Illustrative Example}

Example 5.1. Consider the three-dimensional neural network (2.1) defined by $A=$ $\operatorname{diag}(2,2.4,2.8)$,

$$
\begin{aligned}
B & =\left(\begin{array}{ccc}
-0.25 & -0.1 & 0.15 \\
0.1 & -0.25 & 0 \\
0 & 0.2 & -0.25
\end{array}\right), \quad I(t)=\left(\begin{array}{c}
\sin t \\
-\cos t \\
\sin t
\end{array}\right), \\
g_{i}(\theta) & = \begin{cases}\sqrt{\theta}+1, & \theta>0, \\
0, & \theta=0, \quad i=1,2,3 . \\
0.5 \cos \theta-0.25, & \theta<0,\end{cases}
\end{aligned}
$$

It is easy to see that $g_{i}$ is discontinuous, unbounded, and nonmonotonic and satisfies the assumptions $H_{1}$ and $H_{3} .\|B\|^{2}=0.1712, a=2$. Take $l_{i}=1$ and $P=\operatorname{diag}(1,1,1)$, then $p_{i}=$ $1, \lambda_{m}=0.25$, and we have

$$
l_{i} p_{i}\|B\|^{2}=0.1715<0.25=\frac{1}{2} \lambda_{m} a .
$$




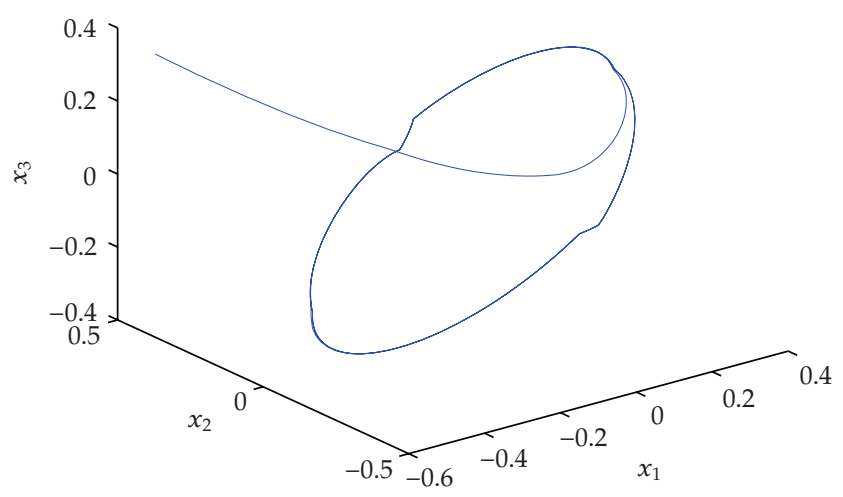

Figure 2: Phase-plane behavior of the state variables $x_{1}, x_{2}$, and $x_{3}$.

All the assumptions of Theorem 4.1 hold and the neural network in Example has a unique $2 \pi$-periodic solution which is globally asymptotically stable.

Figures 1 and 2 show the state trajectory of this neural network with random initial value $(-0.5,0.5,0.3)$. It can be seen that this trajectory converges to the unique periodic solution of this neural network. This is in accordance with the conclusion of Theorem 4.1.

\section{Acknowledgments}

The authors are extremely grateful to anonymous reviewers for their valuable comments and suggestions, which help to enrich the content and improve the presentation of this paper. This work is supported by the National Science Foundation of China (60772079) and the National 863 Plans Projects (2006AA04z212).

\section{References}

[1] M. Di Marco, M. Forti, and A. Tesi, "Existence and characterization of limit cycles in nearly symmetric neural networks," IEEE Transactions on Circuits and Systems I, vol. 49, no. 7, pp. 979-992, 2002.

[2] B. Chen and J. Wang, "Global exponential periodicity and global exponential stability of a class of recurrent neural networks," Physics Letters A, vol. 329, no. 1-2, pp. 36-48, 2004.

[3] J. Cao, "New results concerning exponential stability and periodic solutions of delayed cellular neural networks," Physics Letters A, vol. 307, no. 2-3, pp. 136-147, 2003.

[4] Z. Liu, A. Chen, J. Cao, and L. Huang, "Existence and global exponential stability of periodic solution for BAM neural networks with periodic coefficients and time-varying delays," IEEE Transactions on Circuits and Systems I, vol. 50, no. 9, pp. 1162-1173, 2003.

[5] $\mathrm{H}$. Wu and Y. Li, "Existence and stability of periodic solution for BAM neural networks with discontinuous neuron activations," Computers $\mathcal{E}$ Mathematics with Applications, vol. 56, no. 8, pp. 19811993, 2008.

[6] H. Wu, X. Xue, and X. Zhong, "Stability analysis for neural networks with discontinuous neuron activations and impulses," International Journal of Innovative Computing, Information and Control, vol. 3, no. 6B, pp. 1537-1548, 2007.

[7] $\mathrm{H}$. Wu, "Stability analysis for periodic solution of neural networks with discontinuous neuron activations," Nonlinear Analysis: Real World Applications, vol. 10, no. 3, pp. 1717-1729, 2009.

[8] H. Wu and C. San, "Stability analysis for periodic solution of BAM neural networks with discontinuous neuron activations and impulses," Applied Mathematical Modelling, vol. 33, no. 6, pp. 2564-2574, 2009. 
[9] D. Papini and V. Taddei, "Global exponential stability of the periodic solution of a delayed neural network with discontinuous activations," Physics Letters A, vol. 343, no. 1-3, pp. 117-128, 2005.

[10] $\mathrm{H}$. Wu and X. Xue, "Stability analysis for neural networks with inverse Lipschitzian neuron activations and impulses," Applied Mathematical Modelling, vol. 32, no. 11, pp. 2347-2359, 2008.

[11] H. Wu, J. Sun, and X. Zhong, "Analysis of dynamical behaviors for delayed neural networks with inverse Lipschitz neuron activations and impulses," International Journal of Innovative Computing, Information and Control, vol. 4, no. 3, pp. 705-715, 2008.

[12] H. Wu, "Global exponential stability of Hopfield neural networks with delays and inverse Lipschitz neuron activations," Nonlinear Analysis: Real World Applications, vol. 10, no. 4, pp. 2297-2306, 2009.

[13] M. Forti and P. Nistri, "Global convergence of neural networks with discontinuous neuron activations," IEEE Transactions on Circuits and Systems I, vol. 50, no. 11, pp. 1421-1435, 2003.

[14] M. Forti, P. Nistri, and D. Papini, "Global exponential stability and global convergence in finite time of delayed neural networks with infinite gain," IEEE Transactions on Neural Networks, vol. 16, no. 6, pp. 1449-1463, 2005.

[15] M. Forti, M. Grazzini, P. Nistri, and L. Pancioni, "Generalized Lyapunov approach for convergence of neural networks with discontinuous or non-Lipschitz activations," Physica D, vol. 214, no. 1, pp. 88-99, 2006.

[16] F. H. Clarke, Optimization and Nonsmooth Analysis, Canadian Mathematical Society Series of Monographs and Advanced Texts, John Wiley \& Sons, New York, NY, USA, 1983.

[17] A. F. Filippov, Differential Equations with Discontinuous Right-Hand Side, Mathematics and Its Applications (Soviet Series), Kluwer Academic Publishers, Boston, Mass, USA, 1984.

[18] J.-P. Aubin and A. Cellina, Differential Inclusions: Set-Valued Maps and Viability Theory, vol. 264 of Grundlehren der Mathematischen Wissenschaften, Springer, Berlin, Germany, 1984.

[19] J.-P. Aubin and H. Frankowska, Set-Valued Analysis, vol. 2 of Systems and Control: Foundations and Applications, Birkhäuser, Boston, Mass, USA, 1990.

[20] J. Dugundji and A. Granas, Fixed Point Theory. Vol. I, Monografie Matematyczne, 61, Polish Scientific, Warsaw, Poland, 1982.

[21] N. S. Papageorgiou, "Convergence theorems for Banach space valued integrable multifunctions," International Journal of Mathematics and Mathematical Sciences, vol. 10, no. 3, pp. 433-442, 1987. 